\title{
Lesson from Real-World Experience: Optimal Treatment with Anti- Tumor Necrosis Factor for Ulcerative Colitis
}

\author{
Jaeyoung Chun \\ Department of Internal Medicine, Gangnam Severance Hospital, Yonsei University College of Medicine, Seoul, Korea
}

Corresponding Author

Jaeyoung Chun

ORCID https://orcid.org/0000-0002-4212-0380

E-mail chunjmd@yuhs.ac
See "Real-World Incidence of Suboptimal Response to Anti-Tumor Necrosis Factor Therapy for Ulcerative Colitis: A Nationwide Population-Based Study" by Ju-Young Shin, et al. on page 867 , Vol. 15, No. 6, 2021
Ulcerative colitis (UC) is a chronic systemic inflammatory disorder targeting primarily the gastrointestinal tract and affecting multifocal systemic organs. With no cure, an increased risk of disease flare and malignant processes is associated with uncontrolled inflammation during the disease course of chronic colitis. Patients with UC require lifelong care to prevent or delay disease progression and occurrence of complications, with optimal use of limited therapeutic options available in practice.

The dictionary definition of "optimal" or "optimum" is the best or most favorable results obtainable under special conditions. In the field of medicine, optimal management is the best therapeutic strategy that is expected to produce the most favorable clinical outcomes for patients. Setting therapeutic goals, therefore, is a prerequisite for determining whether a drug use is optimal for the patients with UC. Reducing the need for colectomy seems to be the minimum goals to be achieved in moderate-to-severely active UC requiring biologics or small molecules under consideration of therapeutic target. Quality of life has also been critical as a treat-to-target in patients with UC. ${ }^{1,2}$

The Selecting Therapeutic Targets in Inflammatory Bowel Disease (STRIDE) initiative of the International Organization for the Study of Inflammatory Bowel Diseases (IOIBD) proposed therapeutic targets including clinical/ patient-reported outcome remission (defined as resolution of rectal bleeding and increased stool frequency) and endoscopic remission (defined as a Mayo endoscopic subscore of 0 or 1) for UC. ${ }^{3}$ Recently, the STRIDE-II group has updated the therapeutic targets systematically, according to time required for achieving the goal after starting treat- ment for UC. ${ }^{4}$ Normalization of serum C-reactive protein and fecal calprotectin as well as clinical remission was determined as intermediate targets, and endoscopic healing (defined as a Mayo endoscopic subscore of 0, or UC endoscopic index of severity of 0 or 1 ) is recommended as longterm targets with clinical remission for adult UC patients. ${ }^{4}$ Time to achieve treat-to-target is different based on therapeutic drugs and targets for UC: mean time required for achieving clinical remission and endoscopic healing in UC was 10 and 13 weeks for infliximab, and 11 and 14 weeks for adalimumab, respectively. ${ }^{4}$ Thus, optimal response to anti-tumor necrosis factors (anti-TNFs) for UC should be evaluated by whether the long-term targets were achieved at least 3 months after starting drugs. Taken together, the suboptimal response to anti-TNFs for UC should be determined by the achievement of each therapeutic goal at an appropriate time.

In a population-based cohort study using the unique nationwide claims database of Korea, Shin et al. ${ }^{5}$ reported that $63.5 \%$ of patients with UC experienced suboptimal response within 1 year, and those receiving infliximab (59.1\%) may have a lower rate of suboptimal response compared to adalimumab (69.5\%) or golimumab (68.0\%). Interestingly, the suboptimal response to anti-TNFs was a heterogenous study endpoint including: discontinuation, switching to other biologics, augmentation with other agents, escalation, and colectomy. Other indicators except colectomy did not seem to reflect accurately whether the therapeutic goals for UC were achieved, due to the limitations of the claims database. The most common indicators requiring modification of initial anti-TNF therapy were 
discontinuation, augmentation, and switching to other biologics. Because it could not be defined whether the discontinuation or switching of anti-TNFs was due to adverse events, lack of efficacy, or poor compliance, some patients might decide on the modification of anti-TNF therapy for reasons unrelated to achieving the therapeutic goals required to determine the suboptimal response. Moreover, response to anti-TNF therapy for UC might be optimized if clinical remission and endoscopic healing was achieved by escalation or augmentation with immunomodulators through improvement of pharmacokinetic profile against loss of response.

Notably, the 1-year colectomy rate of patients with UC receiving anti-TNFs was quite low (1.0\%) and similar among anti-TNF agents available in Korea, ${ }^{5}$ in line with previous reports. ${ }^{6,7}$ The major strength of nationwide claims database is to reflect real-world practice. As reported in 2020 inflammatory bowel disease fact sheet in Korea published by the Korean Association for the Study of Intestinal Diseases using the claims data from the National Health Insurance Service, ${ }^{8}$ a proportion of UC patients receiving biologics have been increased up to $8 \%$ steadily in recent decade while colectomy rates seemed to be declining from 2018 in Korea. However, adherence rate to biologics was modest (64\%) and there was a significant difference based on the route of administration $(81 \%$ for infliximab; $37 \%$ for adalimumab; $32 \%$ for golimumab). ${ }^{8}$ Patients with quiescent UC and non-adherence with medication had a 5 -fold greater risk of recurrence, ${ }^{9}$ resulting in failure to achievement of therapeutic goals. Improving adherence for optimal therapeutic strategy with anti-TNF agents in patients with UC is the biggest lesson from the real-world experience.

\section{CONFLICTS OF INTEREST}

J.C. received a grant given by Eisai Co., Ltd. Except for that, no potential conflict of interest relevant to this article was reported.
ORCID

Jaeyoung Chun https://orcid.org/0000-0002-4212-0380

\section{REFERENCES}

1. Jo IH, Lee KM, Kim DB, et al. Quality of life in newly diagnosed moderate-to-severe ulcerative colitis: changes in the MOSAIK cohort over 1 year. Gut Liver. Epub 2021 Aug 11. https://doi.org/10.5009/gnl210141.

2. Keller R, Mazurak N, Fantasia L, et al. Quality of life in inflammatory bowel diseases: it is not all about the bowel. Intest Res 2021;19:45-52.

3. Peyrin-Biroulet L, Sandborn W, Sands BE, et al. Selecting Therapeutic Targets in Inflammatory Bowel Disease (STRIDE): determining therapeutic goals for treat-to-target. Am J Gastroenterol 2015;110:1324-1338.

4. Turner D, Ricciuto A, Lewis A, et al. STRIDE-II: an update on the Selecting Therapeutic Targets in Inflammatory Bowel Disease (STRIDE) initiative of the International Organization for the Study of IBD (IOIBD): determining therapeutic goals for treat-to-target strategies in IBD. Gastroenterology 2021;160:1570-1583.

5. Shin JY, Park HM, Lee MY, Jeon JY, Yoo HJ, Ye BD. Realworld incidence of suboptimal response to anti-tumor necrosis factor therapy for ulcerative colitis: a nationwide population-based study. Gut Liver 2021;15:867-877.

6. Reich KM, Chang HJ, Rezaie A, et al. The incidence rate of colectomy for medically refractory ulcerative colitis has declined in parallel with increasing anti-TNF use: a time-trend study. Aliment Pharmacol Ther 2014;40:629-638.

7. Jenkinson PW, Plevris N, Lyons M, et al. Analysis of colectomy rates for ulcerative colitis in pre- and postbiological eras in Lothian, Scotland. Colorectal Dis 2021;23:1175-1183.

8. Korean Association for the Study of Intestinal Diseases (KASID). 2020 Inflammatory bowel disease fact sheet in Korea [Internet]. Seoul: KASID; c2009 [cited 2021 Nov 02]. Available from: https://www.kasid.org/sub07/IBD_fact_sheet.html.

9. Kane S, Huo D, Aikens J, Hanauer S. Medication nonadherence and the outcomes of patients with quiescent ulcerative colitis. Am J Med 2003;114:39-43. 